\title{
Patient socioeconomic determinants for the choice of the cheapest molecule within a cluster: evidence from Belgian prescription data
}

\author{
France Vrijens • Carine Van de Voorde • \\ Maria-Isabel Farfan-Portet • Robert Vander Stichele
}

Received: 13 April 2011 / Accepted: 21 November 2011 / Published online: 3 December 2011

(C) The Author(s) 2011. This article is published with open access at Springerlink.com

\begin{abstract}
Reference pricing is a common cost-sharing mechanism, with the financial penalty for the use of costly drugs shifted from the third-party payer to the patient. Unintended distributional consequences might arise, if the weakest socioeconomic groups face a relatively higher financial burden. This study analyzed for a sample of Belgian individual prescription data for 4 clusters of commonly used drugs (proton pump inhibitors, statins and two groups of antihypertensives [drugs acting on reninangiotensin system and dihydropyridine derivatives]) whether the probability to receive the least expensive molecule within a cluster was linked to the socioeconomic status of the patient. Logistic regression models included individual demographic, working, chronic illness and financial status and small area education data for 906,543 prescriptions from 1,280 prescribing general practitioners and specialists. For the 4 clusters, results show that patients with lower socioeconomic status consistently use slightly more the least expensive drugs than other patients. Larger effects are observed for patients residing in a nursing home for the elderly, patients entitled to increased reimbursement of co-payments, unemployed, patients treated in a primary care center financed per capita (and not fee-for-service) and patients having a chronic illness. Also, patients residing in neighborhoods with low education status use more less expensive drugs. The findings of the study suggest that although equity considerations were not explicitly taken
\end{abstract}

F. Vrijens · C. Van de Voorde · M.-I. Farfan-Portet ( $₫)$ Belgian Health Care Knowledge Centre (KCE),

Boulevard du Jardin Botanique, 55, 1000 Brussels, Belgium e-mail: maria.farfan@kce.fgov.be

R. Vander Stichele

Heymans Institute of Pharmacology, Medical School, University of Ghent, De Pintelaan 185, 9000 Ghent, Belgium into account in the design of the reference price system, there is no real equity problem, as the costly drugs with supplement are not prescribed more often in patients from lower socioeconomic classes.

Keywords Pharmaceuticals $\cdot$ Reimbursement $\cdot$ Reference pricing $\cdot$ Socioeconomic factors $\cdot$ Cost sharing $\cdot$ Belgium

JEL Classification $\mathrm{H} 51 \cdot \mathrm{I} 11 \cdot \mathrm{I} 18$

\section{Introduction}

Reimbursement rules for outpatient drugs differ from country to country, but all countries face the same challenges: rises in volume and the emergence of new expensive treatments. Faced with increasing costs, third-party payers (TPPs) of the majority of European countries have opted for reference pricing as a method to curb pharmaceutical expenditures [1]. The reference price system (RPS) limits the reimbursement of drugs by establishing a maximum level of reimbursement (the reference price) for a group of pharmaceutical products, called a cluster. In addition to any co-payment(s), the difference between the reference price and the price of a more costly drug has to be paid by the patient. This is usually referred to as the reference supplement. It differs from traditional co-payments on two aspects: First, in general, the supplement can be avoided by a change in prescription (from a drug with supplement to a drug without supplement), and second, it applies to all patients uniformly, no matter their socioeconomic status. Reference pricing is for that reason an example of selective (also called targeted or differential) cost sharing, which intends to provide patients with monetary incentives to alter their consumption behavior. These cost-sharing measures 
are designed in such a way that (part of) the financial incentive and the initiative are shifted from the provider to the demand side and thus expose patients to the financial consequence of their drug use. However, it is not guaranteed that all patients are fully and equally aware of the existence and the consequences of the differentiated amount of cost sharing. Although the general idea behind the cost-sharing designs is to constrain costs, they may have unintended distributional consequences if different socioeconomic groups react differently to the monetary incentives. From a distributional point of view, it is important to know whether the weakest groups in society face a relatively higher financial burden or are treated in a different way because the level of (price) ignorance varies by socioeconomic group. Policies that encourage individual responsibility should therefore be monitored closely to avoid undesirable outcomes in terms of equity. Consequently, differences in use of drugs without supplement by patients of different socioeconomic background should be assessed.

This is precisely the question that this study aims to answer: What are the socioeconomic drivers for the choice of the least expensive molecule (i.e., the choice to prescribe any drug containing this molecule) within a cluster of therapeutically interchangeable molecules? We studied that question for four clusters of commonly prescribed drugs in Belgium: The first cluster relates to proton pump inhibitors (PPI), which are used to treat or prevent gastric reflux and ulcers. The second cluster contains all statins, which are anticholesterol drugs used in primary or secondary prevention of myocardial infarction. The third cluster contains two types of antihypertensives that act on the renin-angiotensin system: the angiotensin conversion inhibitors (ACE) and the angiotensin ii antagonists (sartans). The fourth cluster contains another type of antihypertensive drugs, known as dihydropyridine derivatives. These definitions of a cluster are based on the Anatomical Therapeutical Chemical (ATC) classification of drugs from the WHO [2]: A cluster may contain all drugs belonging to the same pharmacological class (the ATC-4 class, for instance the cluster containing all statins is the C10AA) or may even be more broad and includes drugs having similar therapeutic effects (the ATC-3 class, for instance all drugs that belong to the $\mathrm{C} 09$ class, the agents acting on the renin-angiotensin system). These are typical choices in a therapeutic RPS. In Belgium, a generic RPS for off-patent drugs is in place since 2001 [3, 4], so all drugs belonging to the same cluster based on the ATC-5 class (the level of the molecule) have the same reference price. The choice, in that context, of a generic versus a brand name product is studied in a companion paper [5].

There are, however, signs toward the acceptance of the exchangeability of different molecules within a broader class, which is precisely the hypothesis behind therapeutic reference pricing. One of those signs is the recent measure recommending to all Belgian prescribers to initiate 8 out of 10 new treatments with the least expensive molecule(s) within a pharmacological class of drugs. For instance, for patients starting a statin therapy, physicians had to initiate treatment with either simvastatin or pravastatin, the two molecules with the lowest expenditures per defined daily dose (DDD) for the TPP in 2008.

Before describing the methods and results of our study, we provide a brief overview of the reimbursement of pharmaceuticals in Belgium, with an emphasis of measures aimed at prescribers, and in addition to the details provided in the companion paper [5].

\section{Reimbursement of pharmaceuticals in Belgium}

The amount of the drug expenditures by the TPP is equal to a certain percentage of the drug reimbursement basis. The drug reimbursement basis is the public price for original on-patent drugs and generics and is diminished for original off-patent drugs with a generic alternative on the Belgian market. Coinsurance percentages are adapted regularly and vary from no coinsurance for category A drugs (drugs for life-threatening conditions) to $80 \%$ for category $\mathrm{Cx}$ drugs (reimbursed contraceptives), with the bulk of the reimbursements situated in class B (25\% coinsurance). Coinsurance also differs between patients with and without preferential reimbursement eligibility (basically patients with a low income). ${ }^{1}$

In Belgium, a generic RPS for off-patent drugs was introduced in June 2001, with major modifications in July 2005 and in April 2010. The reference price level is entirely based on a relatively small reduction of the public price of the brand name drug [6] (16\% in 2001, 30\% in 2005 and up to $35 \%$ in 2010 for drugs in the RPS since 4 years). Therefore, a large number of brand drugs companies, when faced with the entry of generic competitors on the market, lowered their price to the reference price level. In that case, patients do not incur any additional reference supplement for brand name drugs and pay only the usual coinsurance. Low-cost drugs, defined as those drugs included in the reference system and not incurring a reference supplement for the patient, are thus generic drugs but also brand name original products that lowered their price to the reference price. However, for patients using brand name drugs that did not lower their prices, accumulation of the reference supplements might become a financial burden. This, in conjunction with the fact that

\footnotetext{
1 The drug cost born by patients changed substantially on April 1, 2010. Since the empirical analysis is based on data for 2008 , we will not elaborate on the new regulation and refer to the website of the TPP for more information (http://www.inami.be/drug/fr/drugs/ general-information/refunding/index.htm\#nature).
} 
Belgium is one of the few European countries denying pharmacists the possibility to deliver generic drugs when brand name drugs are prescribed (the substitution right), may put the patient in a financially difficult situation. Even if the choice of the brand name product is not (entirely) his, he alone has to bear the financial consequence (the reference supplement) of this decision.

This is why measures aimed at influencing prescriber behavior can substantially impact the financial accessibility of the Belgian health care system. These measures are described as follows.

\section{Incentives for physicians to prescribe low-cost drugs}

Minimum percentages of low-cost drug prescriptions (the so-called quotas) were introduced in January 2006. In addition to generic drugs, and to brand name drugs that lowered their price to the reference price level, drugs prescribed under the International Nonproprietary Name (INN) are also considered low cost, whether a generic drug is available or not. This last category has been included because, in case of INN prescription, the pharmacist has to dispense in priority a low-cost drug, if available. Table 1 presents the different quotas by specialty. For instance, general practitioners (GPs), who prescribe $84 \%$ of total DDD in Belgium [7], have to prescribe $27 \%$ of low-cost drugs. Quotas for other specialties range from $9 \%$ for gynecologists to $30 \%$ for gastroenterologists, oncologists, stomatologists and dentists. The quotas were calculated so that, in 2004, half of the physicians within a specialty already reached the quota.

Table 1 Quotas of low-cost prescriptions per specialty

\begin{tabular}{ll}
\hline Specialty & $\begin{array}{l}\text { Quotas of } \\
\text { low-cost } \\
\text { prescriptions } \\
(\%)^{\mathrm{a}}\end{array}$ \\
\hline $\begin{array}{l}\text { Gynecology-obstetric } \\
\text { Pneumology, rheumatology, pediatric, orthopedic }\end{array}$ & $\begin{array}{l}10-14 \\
\text { Neurosurgery, neurology, ophthalmology, ENT, } \\
\quad \text { neuropsychiatry, physical medicine and } \\
\text { rehabilitation, anesthesiology-reanimation, plastic } \\
\text { surgery, urology }\end{array}$ \\
$\begin{array}{l}\text { Dermatology-venereology, psychiatry, surgery, } \\
\text { internal medicine }\end{array}$ & $20-24$ \\
$\begin{array}{l}\text { General medicine } \\
\text { Cardiology, gastroenterology, radiotherapy-oncology, }\end{array}$ & $28-30$ \\
$\quad$ stomatology, dentistry
\end{tabular}

${ }^{a}$ Low-cost drugs include (1) original drugs that have lowered their price to the reference price level, (2) generic drugs, (3) drugs prescribed in International Nonproprietary Name
This quota system undoubtedly rapidly increased the prescription of low-cost drugs: Between 2004 and 2006, respectively, 1 year before and 1 year after the introduction of quotas, the prescription of low-cost drugs, expressed as a percentage of DDD of all reimbursed drugs, increased from 17.2 to $37.1 \%$ [4]. Since then, and because the quotas were not revised since their introduction, the share of low-cost drugs increased very slowly to $40.3 \%$ of DDD prescribed in 2008 [4]. Interestingly, already in September 2006, $97 \%$ of GPs, $85 \%$ of specialists and $93 \%$ of dentists met their quotas of low-cost prescriptions. In 2008, these percentages were unchanged for GPs and dentists, while for specialists they reached $90 \%$ [8]. Implications of not respecting the quotas include close monitoring of the physician prescription pattern, and possibly sanctions, but in practice, this has never been applied.

Beside these quotas, a new measure was introduced in 2009 , which requested physicians to initiate a new treatment with the "least costly molecule" within a cluster of drugs, and this for 4 groups of medications. This choice had to be made for at least 8 of 10 patients, if there were no contraindications and if therapeutic objectives were met. The "least costly molecule" was identified based on TPP expenditure per DDD in 2008. To take into account changes in costs over time, the list of "least costly molecules" per group is updated on a monthly basis.

The four groups of drugs are listed below, with the recommended "least costly molecule":

1. PPI: omeprazole and pantoprazole are recommended.

2. ACE inhibitors and sartans: ACE (any of them) are recommended.

3. Statins: simvastatin and pravastatin are recommended.

4. Nonsteroidal anti-inflammatory drugs (NSAIDs): noncoxibs are recommended.

This study focuses on PPI, statins, ACE and sartans. NSAIDs were not analyzed because the majority of use is over-the-counter (OTC) and is not registered in claims data. We added the class of dihydropyridine derivatives to our study because they are already part of the therapeutic RPS in British Columbia and Germany [9, 10], and we anticipate that Belgian decision-makers could extend the measure to that group of drugs.

\section{Data}

Databases

This study is based on retrospective claims data, available at the individual level. In Belgium, health insurance is 
compulsory, and detailed data on reimbursed pharmaceuticals are available for all patients.

Our data are based on a linkage of three administrative databases, at the individual patient level, which was approved by the Belgian Privacy Commission. ${ }^{2}$

The three administrative databases are:

1. A database containing individual data on patient characteristics of all Belgian sickness funds (Population database) and reimbursed pharmaceutical products delivered by community pharmacists (Pharmanet database), at the detailed level of the prescription (claims data).

2. A health care provider database, which contains information on the year of birth, gender and specialty of the provider.

3. A database of the socioeconomic characteristics of all Belgian inhabitants, aggregated at the level of the statistical sector. Statistical sectors divide municipalities into homogeneous entities in terms of socioeconomic, urban and morphological characteristics. Approximately 20,000 statistical sectors exist in Belgium. They vary in size and are sometimes not larger than a street or a neighborhood.

\section{Sampling procedure}

A sample of the Pharmanet database was available, detailed at the prescription level. A two-step sampling procedure was performed. In a first step, a stratified random sample of $10 \%$ of all prescribing GP and 5\% of all prescribing specialists (SP) was selected in Pharmanet, 2008. The stratification factor was the specialty such as GP, cardiologist, gynecologist (complete list in Table 1). To exclude occasional prescribers (physicians without a practice but who can prescribe for themselves and relatives), prescribers with less than 200 prescriptions in 2008 were not included in the sample (100 prescriptions per semester is the lower limit used by the TPP to include a prescriber in the feedback on low-cost drugs). For all prescribers selected in step 1, all patients who received a prescription from that physician were identified in a second step. Next, all pharmaceutical products delivered in 2008 (in ambulatory setting) to those patients were selected from Pharmanet.

In addition to these data at the individual level, aggregates of the national Pharmanet database were available for the year 2008 to compute expenditures per DDD.

Only patients being at least 18 years old were included in this analysis, because all drugs mentioned above are

\footnotetext{
${ }^{2}$ The authorization can be accessed at http://www.ksz-bcss.fgov. be/nl/bcss/page/content/websites/belgium/security/security_06/ security_06_01.html.
}

indicated for adults (and are given exceptionally to children for very specific indications), and because some important socioeconomic characteristics are defined only for adults (working status is the most important one).

\section{Socioeconomic characteristics}

Patient characteristics available for the analysis were as follows: gender, age, patient is in a rest or nursing home for the elderly, has a guaranteed income, is entitled to increased reimbursement of health services, patient work status (pensioner, invalid and handicapped, unemployed, employee, self-employed), and patient is entitled to a lump sum for chronic illness. The geographical location of the patient residence was also available (Brussels, Wallonia, Flanders).

We also included two variables that are linked to the patient but can also characterize physician behavior. These two variables have in common that they require some patient loyalty to his/her physician in return for financial advantages in primary care. Patients can opt for having a global medical record held by a particular GP. In return, they receive increased reimbursement for their primary care. Patients can also choose to enroll in a primary care center ("maison médicale in French, wijkgezondheidscentrum in Dutch"). Contrary to the majority of GPs who are paid fee-for-service, primary care centers are financed per capita. Patients have free access to the primary care center where they are enrolled (no co-payment) and in return lose their right to get reimbursed if they consult another GP. Being enrolled in a primary care center has no impact on the reimbursement of drugs (co-payment, reference supplement) [11].

Physician characteristics included the specialty (GP or other specialist), gender and age.

Small area information included median taxable income and the education level. Five income groups were created (quintiles). The education variable was defined as the share of individuals having attained post-secondary education, according to the International Standard classification of Education (ISCED) [12], over the total population aged 18 years or more. The implicit hypothesis behind both variables is that high-income or highly educated people have better health literacy $[13,14]$.

\section{Statistical analyses}

First, expenditures in 2008 per DDD were described (TPP expenditures, patient out-of-pocket expenditures and total expenditures) for each molecule for the 4 clusters included in this study: PPI, statins, ACE and sartans, and dihydropyridines derivatives. All molecules available on the 
Belgian market in 2008 for these four classes were included in our analyses. The least costly molecules of each cluster were then based on the expenditures of the TPP. These expenditures are not based on our sample, but represent the national expenditure data from 2008, and were provided by the Belgian National Institute for Health and Disability Insurance (the TPP). However, the number of patients treated by each molecule was calculated based on our sample described above. Second, logistic regression models were fitted to assess associations between patients' and physicians' characteristics and the probability of a patient being treated with the "least costly molecule" within a class. The outcome variable was coded as 1 if the patient received prescriptions for (one of) the "least costly molecule(s)" within a class, and 0 otherwise. The prescriptions from SPs were given twice the weight of those from GPs, to account for differences in sampling ratio. The observation unit was the patient. Patients having received different molecules from the same cluster were not included in the analysis, because we had no insight into the reason underlying this switch (non-response, poor compliance and patient preference). Each patient was then linked to the physician in the sample who prescribed him/ her the largest number of prescriptions. This was done separately for each cluster of drugs.

All factors described in the data section above were included in the final model, whether statistically significant or not. This choice was made to allow for proper comparisons of effects across the four clusters analyzed. Odds ratios and 95\% CI were derived from these regression models. $\mathrm{P}$ values presented are those of the effect of the factor as a whole (i.e., testing whether there is any difference between all levels of the factor), and not $P$ values from pairwise comparisons (testing each level of the factor to a reference category). It is obvious that comparing regression results for 4 clusters of drugs prescribed to many or few patients cannot be solely based on significance of
$P$ values, as the associations of the same magnitude will produce very different $P$ values based only on the size of the sample [15]. Therefore, to allow meaningful comparisons between the 4 clusters, and in addition to results that are statistically significant at $5 \%$, we chose to discuss also results showing at least a $10 \%$ relative difference (OR at least 1.10 , or lower or equal than 0.91 ), being statistically significant or not. This allows comparison of the magnitude of effects across the 4 classes, in addition to the precision of the estimations.

\section{Results}

Selection of prescribers and patients

A total of 1,280 prescribers (having prescribed at least 200 prescriptions in 2008) were selected for this study: 822 GPs (random sample of $10 \%$ of all prescribers) and 458 specialists (stratified sample of 5\% of all prescribers). For these 1,280 prescribers, all prescriptions $(N=906,543$, 94.3\% prescribed by GPs) for their adult patients $(N=203,378,88.0 \%$ treated by GPs) were selected.

Expenditures per DDD and number of patients under the least costly molecules

Tables 2, 3, 4 and 5 present the expenditures per DDD (total, TPP, patient out-of-pocket) and the number of patients in our sample treated by each molecule. The "least costly molecules" of each cluster, used as outcome variable in the regression models, are indicated in bold in the tables.

\section{Proton pump inhibitors (PPI)}

The average expenditure for one DDD of PPI was $€ 0.84$ in 2008: $€ 0.64$ for the TPP and $€ 0.20$ for the patient. Three out

Table 2 Proton pump inhibitors: expenditures per DDD (2008, national data) and the percentage of patients on each molecule (2008, sample of data)

\begin{tabular}{|c|c|c|c|c|c|c|}
\hline \multirow[t]{2}{*}{ ATC5 } & \multirow[t]{2}{*}{ Name } & \multicolumn{3}{|c|}{ Expenditure per DDD $(€)$} & \multirow[t]{2}{*}{$N$ patients in sample } & \multirow[t]{2}{*}{$\%$} \\
\hline & & TPP & Out-of-pocket & Total & & \\
\hline A02BC01 & Omeprazole $^{\mathrm{a}}$ & 0.44 & 0.14 & 0.58 & 45,462 & 67.03 \\
\hline $\mathrm{A} 02 \mathrm{BC} 02$ & Pantoprazole & 1.47 & 0.41 & 1.88 & 11,578 & 17.07 \\
\hline A02BC03 & Lanzoprazole $^{a}$ & 0.52 & 0.22 & 0.74 & 3,282 & 4.84 \\
\hline $\mathrm{A} 02 \mathrm{BC} 04$ & Rabeprazole & 1.10 & 0.84 & 1.94 & 1,697 & 2.50 \\
\hline \multirow[t]{2}{*}{ A02BC05 } & Esomeprazole $^{\mathrm{a}}$ & 1.02 & 0.30 & 1.32 & 5,802 & 8.55 \\
\hline & Total & 0.64 & 0.20 & 0.84 & 67,821 & 100.0 \\
\hline
\end{tabular}

${ }^{a}$ Molecule included in the generic reference price system in 2008. Least costly molecules are indicated in bold

$D D D$ defined daily dose, $T P P$ third-party payer 
Table 3 Statins: expenditures per DDD (2008, national data) and the percentage of patients on each molecule (2008, sample of data)

\begin{tabular}{|c|c|c|c|c|c|c|}
\hline \multirow[t]{2}{*}{ ATC5 } & \multirow[t]{2}{*}{ Name } & \multicolumn{3}{|c|}{ Expenditure per DDD $(€)$} & \multirow[t]{2}{*}{$N$ patients in sample } & \multirow[t]{2}{*}{$\%$} \\
\hline & & ТPP & Out-of-pocket & Total & & \\
\hline C10AA01 & Simvastatin $^{\mathrm{a}}$ & 0.23 & 0.07 & 0.29 & 40,719 & 49.71 \\
\hline C10AA03 & Pravastatin $^{\mathrm{a}}$ & 0.40 & 0.11 & 0.50 & 8,221 & 10.04 \\
\hline C10AA04 & Fluvastatin & 0.57 & 0.15 & 0.72 & 1,285 & 1.57 \\
\hline C10AA05 & Atorvastatin & 1.09 & 0.19 & 1.28 & 19,731 & 24.09 \\
\hline \multirow[t]{2}{*}{ C10AA07 } & Rosuvastatin & 0.66 & 0.15 & 0.81 & 11,959 & 14.60 \\
\hline & Total & 0.56 & 0.12 & 0.68 & 81,915 & 100.0 \\
\hline
\end{tabular}

${ }^{a}$ Molecule included in the generic reference price system in 2008. Least costly molecules are indicated in bold $D D D$ defined daily dose, $T P P$ third-party payer

Table 4 Agents acting on the renin-angiotensin system: expenditures per DDD (2008, national data) and the percentage of patients on each molecule (2008, sample of data)

\begin{tabular}{|c|c|c|c|c|c|c|}
\hline \multirow[t]{2}{*}{ ATC5 } & \multirow[t]{2}{*}{ Name } & \multicolumn{3}{|c|}{ Expenditure per DDD $(€)$} & \multirow[t]{2}{*}{$N$ patients in sample } & \multirow[t]{2}{*}{$\%$} \\
\hline & & TPP & Out-of-pocket & Total & & \\
\hline С09ААО1 & Captopril $^{\mathrm{a}}$ & 0.26 & 0.09 & 0.35 & 1,732 & 2.14 \\
\hline C09AА02 & Enalapril $^{\mathrm{a}}$ & 0.10 & 0.03 & 0.13 & 1,477 & 1.83 \\
\hline C09AA03 & Lisinopril $^{\mathrm{a}}$ & 0.16 & 0.04 & 0.20 & 13,842 & $\mathbf{1 7 . 1 0}$ \\
\hline C09ААО4 & Perindopril $^{\mathrm{a}}$ & 0.40 & 0.11 & 0.51 & 16,160 & 19.97 \\
\hline C09AA05 & Ramipril $^{\mathrm{a}}$ & 0.13 & 0.04 & 0.16 & 7,371 & 9.11 \\
\hline С09ААО6 & Quinapril $^{\mathrm{a}}$ & 0.20 & 0.06 & 0.26 & 1,661 & 2.05 \\
\hline C09AA07 & Benazepril & 0.42 & 0.12 & 0.54 & 3 & 0.00 \\
\hline C09AА08 & Cilazapril & 0.27 & 0.08 & 0.34 & 244 & 0.30 \\
\hline C09AA09 & Fosinopril & 0.55 & 0.16 & 0.71 & 138 & 0.17 \\
\hline С09BА02 & Enalapril and diuretics ${ }^{\mathrm{a}}$ & 0.20 & 0.06 & 0.26 & 568 & 0.70 \\
\hline С09BА03 & Lisinopril and diuretics $^{\mathrm{a}}$ & 0.34 & 0.10 & 0.44 & 4,367 & 5.40 \\
\hline С09BА04 & Perindopril and diuretics & 0.70 & 0.21 & 0.90 & 4,131 & 5.10 \\
\hline C09BA05 & Ramipril and diuretics $^{\mathrm{a}}$ & 0.39 & 0.11 & 0.50 & 249 & 0.31 \\
\hline C09BA05 & Ramipril and felodipine & 0.42 & 0.12 & 0.55 & 467 & 0.58 \\
\hline С09ВА06 & Quinapril and diuretics ${ }^{\mathrm{a}}$ & 0.35 & 0.10 & 0.45 & 80 & 0.10 \\
\hline \multirow[t]{2}{*}{ C09BA08 } & Cilazapril and diuretics & 0.75 & 0.22 & 0.97 & 677 & 0.84 \\
\hline & Subtotal ACE & 0.25 & 0.07 & 0.32 & 53,167 & 65.69 \\
\hline C09CA01 & Losartan & 0.59 & 0.09 & 0.68 & 3,452 & 4.27 \\
\hline C09CA02 & Eprosartan & 0.56 & 0.13 & 0.69 & 1355 & 1.67 \\
\hline C09CA03 & Valsartan & 0.43 & 0.07 & 0.51 & 2,690 & 3.32 \\
\hline C09CA04 & Irbesartan & 0.48 & 0.08 & 0.56 & 2,995 & 3.70 \\
\hline C09CA06 & Candesartan & 0.35 & 0.07 & 0.41 & 2,106 & 2.60 \\
\hline C09CA07 & Telmisartan & 0.43 & 0.07 & 0.50 & 1,947 & 2.41 \\
\hline C09CA08 & Olmesartan medoxomil & 0.42 & 0.09 & 0.52 & 2,056 & 2.54 \\
\hline C09DA01 & Losartan and diuretics & 0.82 & 0.13 & 0.95 & 2,236 & 2.76 \\
\hline C09DA02 & Eprosartan and diuretics & 0.58 & 0.13 & 0.70 & 429 & 0.53 \\
\hline C09DA03 & Valsartan and diuretics & 0.83 & 0.14 & 0.96 & 2,118 & 2.62 \\
\hline C09DA04 & Irbesartan and diuretics & 0.90 & 0.13 & 1.03 & 2,829 & 3.50 \\
\hline C09DA06 & Candesartan and diuretics & 0.68 & 0.13 & 0.81 & 1,537 & 1.90 \\
\hline C09DA07 & Telmisartan and diuretics & 0.86 & 0.13 & 0.99 & 1,503 & 1.86 \\
\hline \multirow[t]{2}{*}{ C09DA08 } & Olmesartan medoxomil and diuretics & 0.53 & 0.13 & 0.66 & 511 & 0.63 \\
\hline & Subtotal sartan & 0.56 & 0.10 & 0.66 & 27,764 & 34.31 \\
\hline
\end{tabular}

${ }^{a}$ Molecule included in the generic reference price system in 2008. Least costly molecules are indicated in bold $D D D$ defined daily dose, $T P P$ third-party payer 
Table 5 Dihydropyridines derivatives: expenditures per DDD (2008, national data) and the percentage of patients on each molecule (2008, sample of data)

\begin{tabular}{|c|c|c|c|c|c|c|}
\hline \multirow[t]{2}{*}{ ATC5 } & \multirow[t]{2}{*}{ Name } & \multicolumn{3}{|c|}{ Expenditure per DDD $(€)$} & \multirow[t]{2}{*}{$N$ patients in sample } & \multirow[t]{2}{*}{$\%$} \\
\hline & & TPP & Out-of-pocket & Total & & \\
\hline C08CA01 & Amlodipine $^{\mathrm{a}}$ & 0.21 & 0.06 & 0.27 & 22,839 & 61.64 \\
\hline C08CA02 & Felodipine $^{\mathrm{a}}$ & 0.20 & 0.09 & 0.29 & 1,243 & 3.35 \\
\hline C08CA03 & Isradipine & 0.54 & 0.15 & 0.70 & 415 & 1.12 \\
\hline C08CA04 & Nicardipine & 0.69 & 0.19 & 0.88 & 88 & 0.24 \\
\hline C08CA05 & Nifedipine $^{\mathrm{a}}$ & 0.30 & 0.12 & 0.42 & 3,486 & 9.41 \\
\hline C08CA07 & Nisoldipine & 0.86 & 0.24 & 1.09 & 883 & 2.38 \\
\hline C08CA08 & Nitrendipine & 0.65 & 0.18 & 0.83 & 52 & 0.14 \\
\hline С08CA09 & Lacidipine & 0.55 & 0.15 & 0.70 & 592 & 1.60 \\
\hline C08CA12 & Barnidipine & 0.39 & 0.11 & 0.51 & 3,092 & 8.35 \\
\hline \multirow[t]{2}{*}{ C08CA13 } & Lercanidipine & 0.41 & 0.12 & 0.53 & 4,360 & 11.77 \\
\hline & Total & 0.27 & 0.08 & 0.35 & 37,050 & 100.0 \\
\hline
\end{tabular}

${ }^{a}$ Molecule included in the generic reference price system in 2008. Least costly molecules are indicated in bold $D D D$ defined daily dose, $T P P$ third-party payer

of 5 PPI were included in the RPS: omeprazole, lanzoprazole and esomeprazole. Omeprazole had the lowest expenditure per DDD ( $€ 0.58$ per DDD) and was the most used drug (67\% of patients). Lanzoprazole had the second lowest expenditure per DDD (€0.74 per DDD) and was used by a small fraction of patients $(4.8 \%)$. Esomeprazole $(€ 1.32$ per DDD) had no generic version available, but was nevertheless included in the RPS due to its nature of isomer of omeprazole. The two molecules with the highest expenditures were pantoprazole ( $€ 1.88$ per DDD, $17 \%$ of patients) and rabeprazole ( $€ 1.94$ per DDD, $8.6 \%$ of patients).

\section{Statins}

The molecule with the lowest expenditure, simvastatin (€0.29 per DDD), was used by half of the patients $(49.7 \%)$. The molecule with the second lowest expenditure, pravastatin ( $€ 0.50$ per DDD), was used by $10 \%$ of patients. For fluvastatin ( $€ 0.72$ per DDD, $1.6 \%$ of patients), rosuvastatin (€0.81 per DDD, $14.6 \%$ of patients) and atorvastatin (€1.28 per DDD, $24 \%$ of patients), there was no generic alternative in 2008, and hence, they were not part of the generic reference price system.

\section{Agents acting on the renin-angiotensin system (ACE and sartans)}

Agents acting on the renin-angiotensin system include angiotensin conversion enzyme inhibitors (ACE) and angiotensin ii antagonists (sartans). The total expenditure per DDD was $€ 0.32$ for ACE (used by $66 \%$ of patients) and $€ 0.67$ for sartans (used by $34 \%$ of patients). There is, however, a large variability in expenditure per DDD in the group of cheap molecules (the ACE), resulting that some molecules from that group have higher expenditure than some of the molecules from the expensive group (the sartans). This happens because this cluster combines plain treatment (ACE or sartan) and plain treatment associated with diuretics, which are generally more expensive.

\section{Dihydropyridines derivatives}

There were 11 molecules on the Belgian market within the class of dihydropyridines derivatives, three of which had a low-cost alternative and were included in the reference price system. Amlodipine, the molecule with the lowest expenditure per DDD ( $€ 0.27$ per DDD), was also the most used $(67.6 \%$ of patients).

Influence of patients' and physicians' socioeconomic characteristics

Table 6 presents the results of the logistic regressions for the 4 clusters of drugs. Demographic patient characteristics do influence the probability of being prescribed the least costly molecule within a cluster, but results are contrasted across the 4 clusters. The strongest effect of age is seen for the ACE and sartan group, where patients above 45 years old receive less cheap molecules than younger patients. This is also true for the class of PPI, where patients above 45 years old consistently receive less omeprazole and pantoprazole than patients below 45 years old. For the class of statins and dihydropyridine derivatives, there is a statistical association with age, but the association is not 


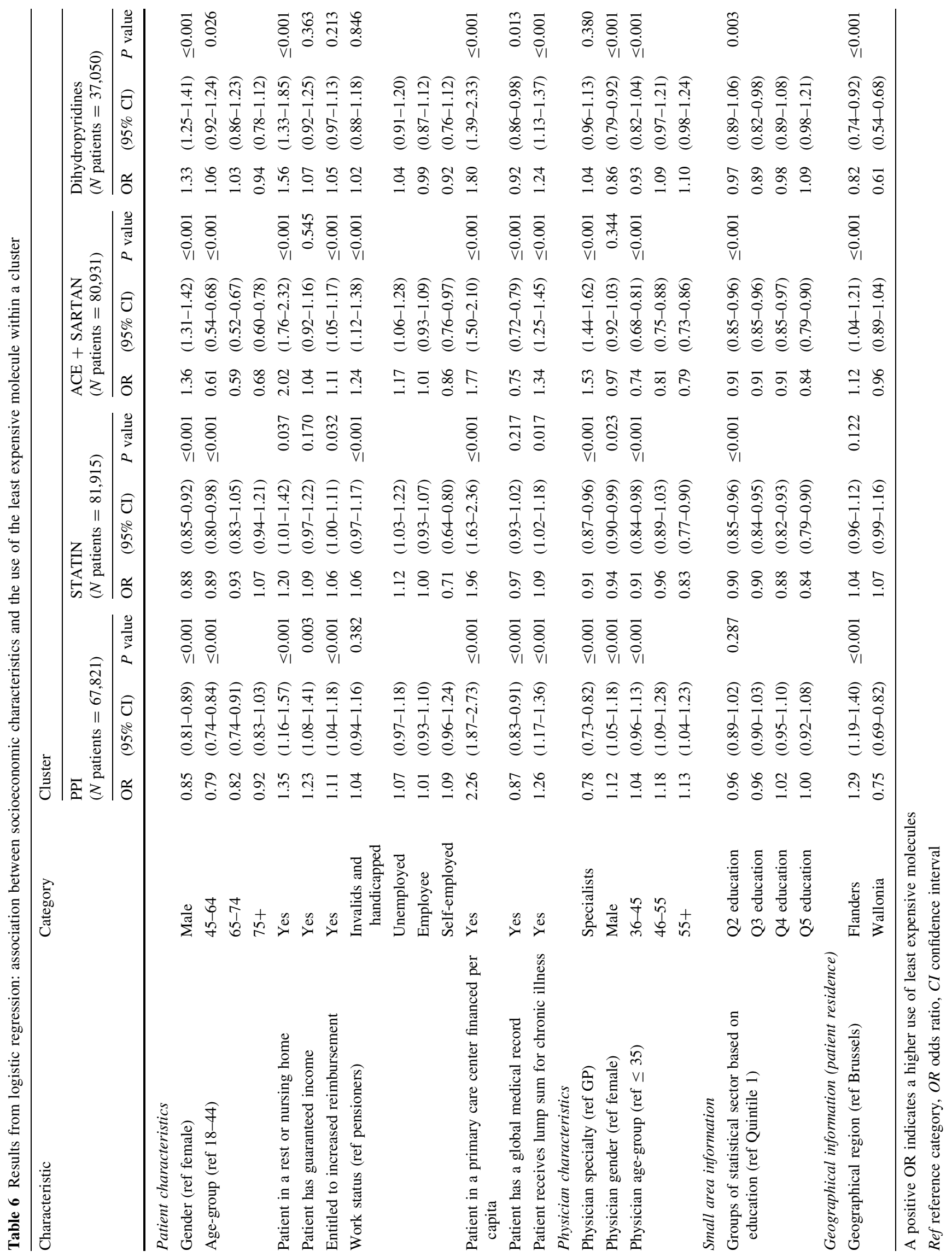


monotonous. However, for the 4 clusters, a very strong and consistent association is found with elderly patients in a rest or nursing home: They are more likely to receive the least costly molecule within the cluster. Patient gender also influences this probability: Male patients receive more of those drugs for the two antihypertensive classes, but less for the statins and PPIs.

As far as socioeconomic characteristics are concerned, all indicators point to the same direction, namely that patients having a lower socioeconomic status are prescribed more least costly molecules. Patients being entitled to a guaranteed income and patients eligible for increased reimbursement of co-payments have a higher probability of receiving the least costly molecule in the group than patients without guaranteed income or increased reimbursement (all OR are positive), with the largest effects seen for the PPI class. For the work status, patients not working (invalids or handicapped and unemployed) consistently use more least costly molecules within a cluster than employees. The strongest effects are seen for the ACE and sartans cluster. Self-employed patients also seem to use more costly molecules than employees, except for PPI.

A very strong and consistent effect was found for those patients belonging to a primary care center financed per capita; they receive for all 4 clusters more of the least costly molecules than other patients. Although we defined this variable as a patient characteristic, very probably, the effect is the result of specific patient and physician characteristics. Surprisingly, holding a global medical record is associated with lower use of cheap molecules for PPI, ACE and sartan and dihydropyridines.

Patients entitled to a lump sum for chronic illness receive more least costly molecules than those who are not entitled. This result is consistent across the 4 clusters.

Physician characteristics also influence the prescription of least costly molecules. GPs prescribe more least costly molecules for PPI and statins. The reverse is true for ACE/ sartans. For dihydropyridine derivatives, there is no association with specialty. Physician gender and age are also associated with the prescription of a least costly molecule, but there are no consistent patterns across the 4 clusters: For the statins and ACE/sartans, older physicians prescribe less least costly molecules. The reverse is true for PPI.

Analysis of the model robustness revealed collinearity problems between the two small area characteristics, income and education. Based on sensitivity analyses, it was decided to exclude the income variable from the model (tables including income are available from the authors). Education has a strong effect for two clusters: Patients under statin or ACE/sartan living in small areas with low education levels are more likely to receive cheap molecules, and this effect is increasing with the level of education. For the two other drug classes, there are no clear patterns.
Odds ratios from the geographical location of the patient show that, after adjustment for demographic, socioeconomic and small area information, there are still strong differences in the use of the least expensive molecules within clusters, except for the statin group. Patients living in Flanders have more chance to receive the least expensive molecule than patients living in Brussels for the PPI cluster for the ACE + sartan cluster, but the reverse holds for the class of dihydropyridines.

\section{Discussion}

When examining the RPS from the point of view of financial accessibility of the health care system, a possible differential impact of the RPS on persons with a different socioeconomic background should be assessed. This study shows reassuring results, as no evidence was found that patients of lower socioeconomic status use more expensive molecules when cheaper alternatives exist within a cluster of drugs.

These results are in line with the very scarce studies that have already been published on that subject. In order to find the relevant empirical evidence, we conducted electronic searches in the most common databases (see search algorithm in appendix). Most studies concentrated on adults aged 65 and older living in British Columbia (Canada), which has a therapeutic RPS. It is striking that we could only find 3 studies that studied the differentiated impact of therapeutic RPS on socioeconomic groups. We briefly describe those 3 studies, which analyze 2 groups of drugs: ACE and calcium channel blockers.

The first two studies, from the same authors, assessed the impact of the introduction of therapeutic RPS for ACEs [16] and for calcium channel blockers (CCB) [9] in British Columbia and explored the association with socioeconomic status (based on their income). Conclusions of both studies converge: First, low-income patients have a higher probability of stopping medications than high-income patients, but this is true even before introduction of the RPS. Second, compared to high-income patients, those on low and middle income were more likely to switch from the expensive drug (having a reference supplement) to a drug without supplement or to switch to another antihypertensive therapy. A third study [17] focused on the effect of physician gender on changes in prescribing patterns of ACE, also in British Columbia. The authors found that patients of female physicians were more likely to remain on cost-sharing ACE inhibitors with an exemption. The authors argued that this difference might be related to the fact that female physicians are more responsive to their patients' requests.

Our results are in line with those few studies from British Columbia: Economically frail patients seem to use 
the least expensive molecules within a cluster and bear a lower financial burden for their medications than patients of higher socioeconomic status. Nevertheless, as our findings are based on observational data, they have to be read cautiously in view of some limitations. First, we based our analysis on consumers only (patients who were prescribed reimbursed medications), and not on the total Belgian population. The implicit unproven hypothesis behind this choice is that all patients needing treatment have access to it. A second limitation is that the availability of socioeconomic variables in the sickness funds database is rather limited; especially, the lack of information on education at the individual level is a drawback. This constraint was partially offset by the use of the education level available for the place of residence of the patient. Other limitations were about the design of the study itself. We had only access to a proportionally smaller sample for specialist prescriptions (sample of 5\%) compared to GPs (sample of $10 \%$ ). This implies that our results for the specialists are less reliable than our results for the GPs. Also, we could only link each patient to the most prescribing physician within our sample of physicians, and not in absolute terms. We are thus not sure to have captured the most important patient-physician relationship for all patients.

One strength of the study is the linkage of three databases (one for patient characteristics, one for physician characteristics and one for small area characteristics) combined with a large sample size (more than 900,000 prescriptions in 2008), which makes the data particularly suited for the specific research question in this paper.

There is, to date, no therapeutic RPS in Belgium. The assessment in the study of possible unintended distributional consequences of that kind of system identified no systematic differences in the use of the least costly molecules against less privileged socioeconomic groups. This is a reassuring message for health decision-makers who intend to enlarge the current selection of groups to other pharmacological classes and other types of users (removing the current restriction to the new users target population). This result may be due to the fact that poor patients react to monetary signals and interact with physicians who are well informed on this matter. It may also be the case that physicians take their quotas seriously and have actively selected low-cost medicines, especially for their deprived patients. Indeed, Belgium's health care system has historically aimed to guarantee access to health services for vulnerable population groups. A good example of a measure in line with this objective is the early establishment of the TPP system that allows a direct payment to the pharmacist of the reimbursable part of any delivered drug. In conclusion, introducing a selective cost-sharing measure should be accompanied by measures guaranteeing equal access to information on prices and therapies, as a necessary step to avoid inequities among patients.

Acknowledgments The authors wish to thank Marc de Falleur (Belgian National Institute for Health and Disability Insurance) for providing the national expenditure data for 2008, Maité le Polain for her help on describing the Belgian reimbursement system and Erik Schokkaert for his comments on the first draft of this manuscript. The authors also wish to thank both referees for their helpful comments.

Open Access This article is distributed under the terms of the Creative Commons Attribution Noncommercial License which permits any noncommercial use, distribution, and reproduction in any medium, provided the original author(s) and source are credited.

\section{Appendix: Algorithm for the electronic search of relevant articles}

In order to find the relevant empirical evidence, we conducted electronic searches in Medline, Embase, Econlit and in the Cochrane Library database for studies published between 1988 and 2009.

The search was based on a set of key words (MeSH terms and/or text words): reference pricing OR reference price OR reference prices OR reference based pricing OR reference adj2 pric $\$$.

We defined a priori eligibility criteria (the study had to be based on empirical analysis and had to examine the effects of a reference price system on drug use and/or health outcomes and/or costs), which led to the selection of 35 relevant articles.

Of those, only three studies discussed the effect of patient socioeconomic characteristics. The results of those three studies are described in the main text.

\section{References}

1. Aaserud, M., Dahlgren, A.T., Kosters, J.P., Oxman, A.D., Ramsay, C., Sturm, H.: Pharmaceutical policies: effects of reference pricing, other pricing, and purchasing policies. Cochrane Database Syst. Rev. 2(2), CD005979 (2006)

2. World Health Organization: ATC Structure and principles (2010)

3. Simoens, S., De Bruyn, K., Bogaert, M., Laekeman, G.: Pharmaceutical policy regarding generic drugs in Belgium. Pharm. Econ. 23(8), 755-766 (2005)

4. Vrijens, F., Van de Voorde, C., Farfan-Portet, M.I., le Polain, M., Lohest, O.: The reference price system and socioeconomic differences in the use of low cost drugs. In: Health Services Research (HSR), vol. 126. Belgian Health Care Knowledge Centre (KCE), Brussels (2010)

5. Farfan-Portet, M.I., Van de Voorde, C., Vrijens, F., Vander Stichele, R.: Patient socioeconomic determinants of the choice of generic versus brand name drugs in the context of a reference price system: evidence from Belgian prescription data. Submitted as companion paper (2011) 
6. Adriaen, M., De Witte, K., Simoens, S.: Pricing strategies of originator and generic medicines following patent expiry in Belgium. Journal of Generic Medicines 5(3), 175-187 (2008)

7. National Institute for Health and Disability Insurance (RIZIV/ INAMI): [Drugs deliveries in ambulatory sector]. http://www. inami.fgov.be/drug/fr/statistics-scientific-information/pharmanet/ pharmaceutical-tables/pdf/2007/tables2007.pdf (2007)

8. National Institute for Health and Disability Insurance (RIZIV/ INAMI): [Prescription of a minimum percentage of low cost medications_feedback 2009]. http://www.inami.be/care/fr/ doctors/promotion-quality/feedbacks/feedback-generica/feedback 2009.htm (2010)

9. Schneeweiss, S., Soumerai, S.B., Maclure, M., Dormuth, C., Walker, A.M., Glynn, R.J.: Clinical and economic consequences of reference pricing for dihydropyridine calcium channel blockers. Clin. Pharmacol. Ther. 74(4), 388-400 (2003)

10. The National Association of Statutory Health Insurance Funds [reference prices for medicines]. http://www.gkv-spitzenverband. de/upload/Übersicht_Festbetragsfestsetzungen_091106_10031. pdf (2009)

11. Annemans, L., Closon, J.-P., Closon, M.-C., Heymans, I., Lagasse, R., Mendes da Costa, E., Moureaux, C., Roch, I.: Comparison of the cost and the quality of two financing systems of primary health care in Belgium. In: Health Services Research (HSR), vol. 85. Belgian Health Care Knowledge Centre (KCE), Brussels (2008)

12. Unesco: International Standard Classification of Education (ISCED)—1997 In. Unesco (2006)

13. Volandes, A.E., Paasche-Orlow, M.K.: Health literacy, health inequality and a just healthcare system. Am J Bioethics AJOB 7(11), 5-10 (2007)

14. Grossman, M.: On the concept of health capital and the demand for health. J. Polit. Econ. 80(2), 223 (1972)

15. Stang, A., Poole, C., Kuss, O.: The ongoing tyranny of statistical significance testing in biomedical research. Eur. J. Epidemiol. 25(4), 225-230 (2010)

16. Schneeweiss, S., Soumerai, S.B., Glynn, R.J., Maclure, M., Dormuth, C., Walker, A.M.: Impact of reference-based pricing for angiotensin-converting enzyme inhibitors on drug utilization. Can. Med. Assoc. J. 166(6), 737-745 (2002)

17. Duetz, M.S., Schneeweiss, S., Maclure, M., Abel, T., Glynn, R.J., Soumerai, S.B.: Physician gender and changes in drug prescribing after the implementation of reference pricing in British Columbia. Clin. Therap. 25(1), 273-284 (2003) 